Revue Française de Civilisation Britannique

French Journal of British Studies

XII-2 | 2003

La situation et les politiques de l'emploi en France et en Grande-Bretagne, 1990-2000

\title{
Vers une nouvelle géographie du chômage?
}

Towards a New Geography of Unemployment?

\section{Victor Sarafian}

\section{OpenEdition}

Journals

Édition électronique

URL : http://journals.openedition.org/rfcb/3102

DOI : $10.4000 /$ rfcb.3102

ISSN : 2429-4373

Éditeur

CRECIB - Centre de recherche et d'études en civilisation britannique

\section{Édition imprimée}

Date de publication : 1 avril 2003

ISBN : 2-911580-16-8

ISSN : 0248-9015

Ce document a été généré automatiquement le 31 mai 2019.

\section{(c)}

Revue française de civilisation britannique est mis à disposition selon les termes de la licence Creative Commons Attribution - Pas d'Utilisation Commerciale - Pas de Modification 4.0 International. 


\title{
Vers une nouvelle géographie du chômage?
}

\author{
Towards a New Geography of Unemployment?
}

\author{
Victor Sarafian
}

$1 \mathrm{Au}$ cours des années quatre-vingt-dix, on a assisté à une modification profonde des disparités régionales en Grande-Bretagne, qui prennent traditionnellement la forme d'un déséquilibre entre le Nord et le Sud du pays. La ligne de partage représentée par un tracé imaginaire allant de l'estuaire de la Severn, au sud du pays de Galles, à celui de la Humber, au sud du Humberside, sépare les régions du sud à savoir le Sud-Est, le SudOuest, l'Est Anglie et les Midlands de l'Est, des régions du nord : l'Écosse, le Nord, le Yorkshire \& Humberside, le Nord-Ouest, le pays de Galles et les Midlands de l'Ouest. Le clivage traditionnel en matière de chômage, qui avait tant marqué les années quatrevingt, s'est beaucoup atténué pendant la décennie suivante. Cette convergence des dynamiques régionales a surpris la plupart des observateurs car historiquement, en Grande-Bretagne, les disparités régionales tendent à se renforcer en période de récession. Or, si la récession du début des années quatre-vingt s'est effectivement traduite par une aggravation du clivage Nord-Sud, celle du début des années quatre-vingt-dix s'est accompagnée d'une réduction sensible de l'écart observé dans la décennie précédente.

2 Au début des années quatre-vingt-dix, on annonce, ici et là, l'apparition d'une nouvelle géographie du chômage et la fin du clivage Nord-Sud. Ian Hamilton Fazey, journaliste au Financial Times, parle ainsi de cette nouvelle donne géographique :

...quelque chose d'étrange est en train de se produire par rapport au clivage NordSud... Alors qu'au cours des années quatre-vingt on était accablé par le fossé qui s'élargissait entre le Nord en déclin et le Sud prospère, aujourd'hui les rôles, semble-t-il, sont en train de s'inverser. ${ }^{1}$

3 A la lecture du graphique ci-après, qui montre l'évolution de l'écart du taux de chômage entre le Nord et le Sud par rapport à la moyenne nationale, on constate effectivement que les taux se resserrent considérablement au cours des années quatre-vingt-dix. La trajectoire des deux courbes prend la forme d'une «bouteille »; le taux de chômage dans le Nord demeure légèrement plus élevé que dans le Sud, mais la différence entre les deux 
parties du pays n'est plus aussi notable qu'elle l'a été pendant les années quatre-vingt. En effet, en 1995 le taux de chômage dans le Nord était à peine de 12\% supérieur au taux de chômage du Sud, alors que pendant la décennie précédente, le chômage dans le Nord avait atteint un taux allant jusqu'à $97 \%$ de plus que dans le Sud.

Graphique 1. Écart du taux de chômage entre le Nord et le Sud, 1980-1995

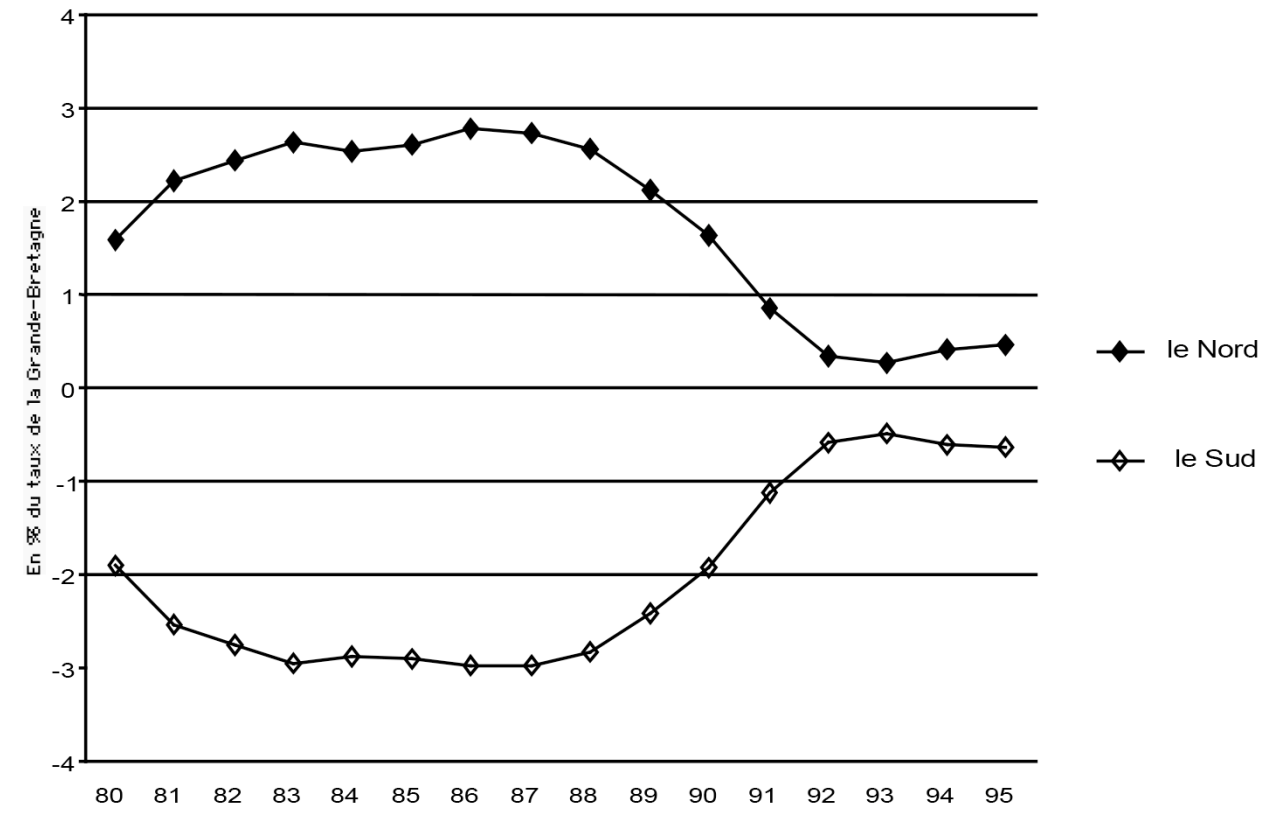

Source : graphique établi par l'auteur d'après Regional Trends, 1989 p. 120 ; 1996 p. 90 l'incidence du chômage, on est ainsi passé à une situation de convergence et d'atténuation de celles-ci. Cet article s'efforce de donner quelques éclairages sur l'apparition de la « nouvelle » géographie du chômage.

5 Tout d'abord, il convient de signaler que lorsque les disparités régionales se sont aggravées pendant les années quatre-vingt, les articles et les ouvrages sur la question se sont effectivement multipliés, mais curieusement, lorsque le clivage Nord-Sud s'est atténué aux cours des années quatre-vingt-dix, les études consacrées à la compréhension de cette nouvelle donne ont été beaucoup moins nombreuses. La configuration territoriale du chômage résulte d'un jeu complexe de facteurs; nous nous limiterons ici à éclaircir certaines caractéristiques de cette nouvelle donne géographique, en analysant d'abord l'évolution de la structure du chômage par sexe, classe sociale et durée, puis en examinant la question des liens entre chômage, emploi et inactivité.

\section{Le clivage Nord-Sud : une « affaire d'hommes »}

Curieusement, les disparités régionales des taux de chômage analysées sous l'angle des différences entre hommes et femmes sont un sujet largement ignoré des chercheurs. La question présente pourtant un intérêt certain, car elle permet de mieux comprendre pourquoi le clivage Nord-Sud et les disparités régionales se sont atténués. En effet, la convergence entre le Nord et le Sud repose avant tout sur la diminution des écarts du taux de chômage féminin. Comme on peut le constater à travers le graphique suivant, à

Revue Française de Civilisation Britannique, XII-2 | 2003 
partir du début des années 1990, les écarts chez les femmes se réduisent sensiblement, à tel point qu'en 1995 les femmes du Nord et celles du Sud ont le même taux de chômage. Entre les hommes, on remarque également une réduction des écarts, mais elle est nettement moins marquée que chez les femmes.

Graphique 2. Taux de chômage dans le Nord et le Sud par sexe, 1980-1995

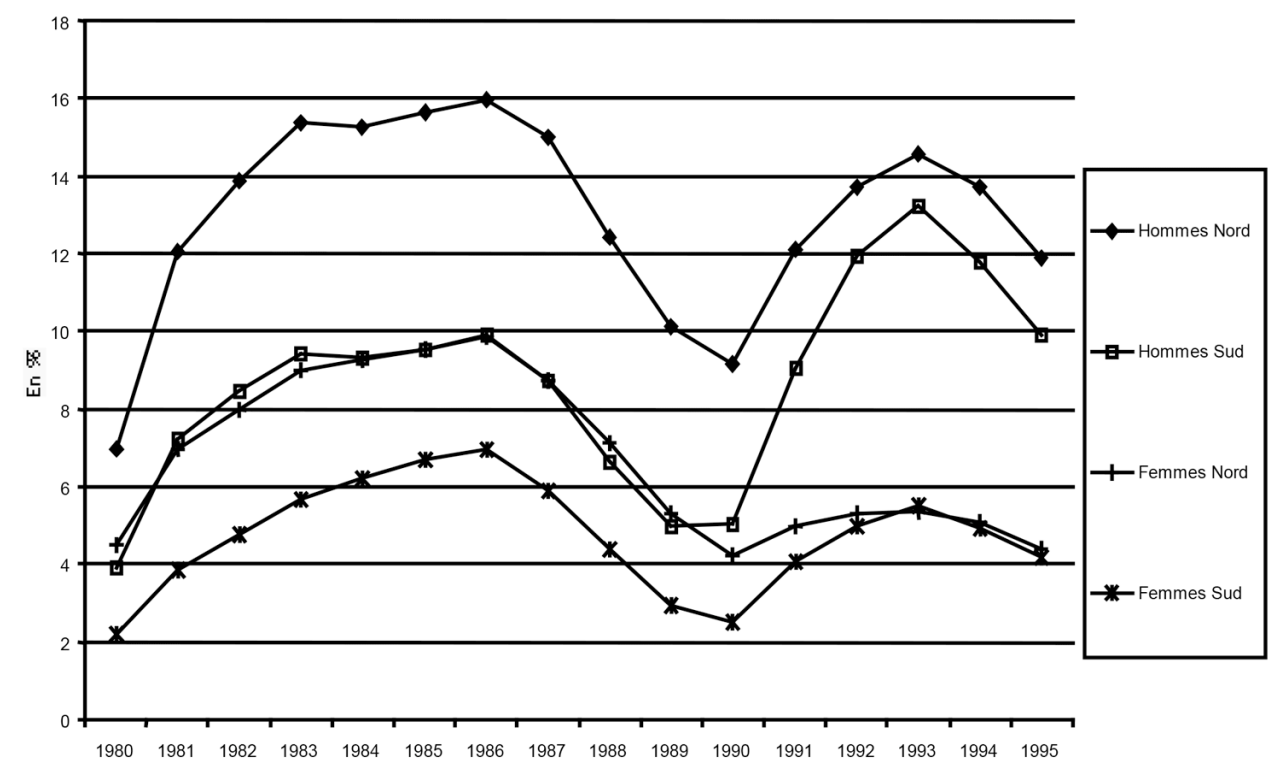

Source : graphique établi par l'auteur d'après "Seasonally adjusted claimants", via Nomis

7 À l'origine de ce rapprochement, il faut mettre en avant la hausse particulièrement faible du chômage des femmes dans les régions du Nord pendant la récession du début des années 1990. Comment donc expliquer ce fait?

8 Pour répondre, il convient de rappeler ce qui a caractérisé la récession du début des années 1990 par rapport à celle du début des années 1980. Une idée souvent avancée pour expliquer la nouvelle géographie du chômage consistait à dire que la récession du début des années 1990, contrairement à celle du début des années 1980, avait frappé davantage le secteur des services. L'économie du Sud, plus dépendante de ce secteur, en avait donc subi plus fortement les contrecoups. Cette hypothèse n'est pas tout à fait exacte car tout comme lors de la première récession, c'est dans le secteur industriel que les plus grandes pertes d'emplois ont été enregistrées (voir tableau ci-après). En réalité, ce qui a caractérisé la récession du début des années quatre-vingt-dix, c'est le fait qu'au Nord, l'industrie a fait non seulement «jeu égal » avec l'industrie du Sud, mais surtout, que l'emploi dans les services a progressé au Nord, alors qu'il a diminué au Sud. La progression de l'emploi dans les services ne pouvait qu'être à l'avantage des femmes, ce secteur étant nettement plus féminisé. 
Tableau 1. Évolution de l'emploi dans le secteur industriel et des services, 1980-83 et 1990-93

\begin{tabular}{|c|c|c|c|c|}
\hline & \multicolumn{4}{|c|}{ Progression de l'emploi en \% } \\
\hline & Industrie & Services & Industrie & Services \\
\hline & $1980-83$ & $1980-83$ & $1990-93$ & $1990-93$ \\
\hline Nord & $-22,2$ & $-5,5$ & $-15,6$ & 2,7 \\
\hline Yorkshire and Humberside & $-19,9$ & $-3,0$ & $-11,8$ & 5,7 \\
\hline Midlands de l'Ouest & $-22,7$ & $-4,2$ & $-14,6$ & 0,3 \\
\hline Nord-Ouest & $-22,2$ & $-2,6$ & $-22,5$ & 0,3 \\
\hline Pays de Galles & $-23,3$ & $-1,2$ & $-17,2$ & 0,1 \\
\hline Écosse & $-18,4$ & $-0,9$ & $-14,2$ & 4,8 \\
\hline Total Nord & $-21,4$ & $-2,8$ & $-16,3$ & 2,4 \\
\hline Midlands del'Est & $-15,6$ & $-2,0$ & $-11,1$ & $-0,3$ \\
\hline Est Anglie & $-13,6$ & 3,4 & $-6,4$ & 2,6 \\
\hline Sud-Est & $-13,3$ & $-1,7$ & $-19,9$ & $-11,4$ \\
\hline Sud-Ouest & $-15,8$ & $-0,5$ & $-17,7$ & 0,8 \\
\hline Total Sud & $-14,1$ & $-1,3$ & $-16,7$ & $-7,7$ \\
\hline Grande-Bretagne & $-18,2$ & $-2,0$ & $-16,5$ & $-3,3$ \\
\hline
\end{tabular}

Source : tableau établi par l'auteur d'après Employment Gazette, via Nomis 2001.

9 Si le problème régional est ainsi devenu essentiellement une " affaire d'hommes ", il ne signifie pas pour autant que les conditions d'emploi des femmes sont devenues plus favorables, notamment en matière de salaire, de précarité et de sous-emploi. ${ }^{2}$

\section{Le clivage Nord-Sud : un problème de classe sociale}

10 Le clivage Nord-Sud a bien sûr été aussi une affaire de classes sociales. En GrandeBretagne, comme dans la plupart des pays industrialisés, le taux de chômage augmente au fur et à mesure que l'on descend dans la hiérarchie sociale. Les profils socioprofessionnels de la main-d'œuvre peuvent varier d'une région à une autre. De ce fait, on peut s'interroger sur les liens entre l'atténuation du clivage Nord-Sud et l'évolution de la composition socioprofessionnelle des régions. Curieusement encore, cette question a été peu étudiée. Pourtant, elle permet de préciser certaines caractéristiques de la nouvelle géographie du chômage.

11 En effet, si on examine les taux de chômage régionaux par groupes socioprofessionnels, on constate que le déséquilibre traditionnel entre le Nord et le Sud repose essentiellement sur les différences entre les groupes les moins qualifiés. Le tableau ciaprès montre les taux de chômage régionaux par classes sociales ainsi que les taux de chômage des travailleurs manuels et non manuels. Les six classes sociales correspondent à celles définies par les autorités britanniques lors du recensement de la population. Les groupes I à $\mathrm{V}$ vont du plus qualifié au moins qualifié, à savoir : I (cadres supérieurs et professions libérales), II (professions intermédiaires), III N (travailleurs non manuels qualifiés) III M (travailleurs manuels qualifiés) IV (travailleurs manuels semi-qualifiés) et $\mathrm{V}$ (travailleurs manuels non qualifiés). 
Tableau 2. Taux de chômage régional par classe sociale, travailleurs manuels et non manuels

\begin{tabular}{|c|c|c|c|c|c|c|c|c|c|c|c|c|c|c|c|c|}
\hline & \multicolumn{8}{|c|}{1981} & \multicolumn{8}{|c|}{1991} \\
\hline & \multicolumn{6}{|c|}{ Classe Sociale } & \multirow[b]{2}{*}{$\begin{array}{c}\text { Non } \\
\text { Manuel }\end{array}$} & \multirow[b]{2}{*}{ Manuel } & \multicolumn{6}{|c|}{ Classe Sociale } & \multirow[b]{2}{*}{$\begin{array}{c}\text { Non } \\
\text { Manuel }\end{array}$} & \multirow[b]{2}{*}{ Manuel } \\
\hline & 1 & $\|$ & IIIN & IIIM & IV & $\mathrm{v}$ & & & 1 & ॥ & IIIN & IIIM & IV & $\mathrm{v}$ & & \\
\hline Grande-Bretagne & 3 & 3 & 4 & 10 & 10 & 15 & 4 & 10 & 3 & 4 & 5 & 9 & 10 & 13 & 4 & 10 \\
\hline "Le Sud" & 2 & 3 & 4 & 7 & 7 & 11 & 3 & 8 & 3 & 4 & 5 & 9 & 9 & 12 & 4 & 9 \\
\hline "Le Nord" & 3 & 3 & 5 & 12 & 12 & 18 & 4 & 13 & 3 & 4 & 5 & 10 & 11 & 14 & 4 & 11 \\
\hline Sud-Est & 2 & 3 & 3 & 7 & 7 & 10 & 3 & 7 & 3 & 4 & 6 & 9 & 10 & 12 & 5 & 10 \\
\hline Est Anglie & 2 & 3 & 4 & 7 & 7 & 12 & 3 & 8 & 3 & 3 & 4 & 7 & 8 & 10 & 4 & 8 \\
\hline Sud-Ouest & 3 & 4 & 4 & 8 & 8 & 11 & 4 & 8 & 3 & 4 & 5 & 9 & 9 & 12 & 4 & 9 \\
\hline Midlands de l'Est & 2 & 3 & 4 & 8 & 8 & 13 & 3 & 9 & 3 & 4 & 5 & 9 & 9 & 12 & 4 & 9 \\
\hline Midlands de l'Ouest & 3 & 3 & 5 & 12 & 11 & 14 & 4 & 12 & 2 & 4 & 5 & 9 & 10 & 12 & 4 & 10 \\
\hline Yorkshire \& Humberside & 3 & 3 & 4 & 10 & 10 & 16 & 4 & 11 & 2 & 4 & 5 & 10 & 11 & 14 & 4 & 11 \\
\hline Nord-Ouest & 2 & 4 & 6 & 12 & 12 & 20 & 4 & 13 & 2 & 4 & 5 & 10 & 12 & 16 & 4 & 12 \\
\hline Nord & 3 & 4 & 6 & 13 & 13 & 21 & 5 & 14 & 3 & 4 & 5 & 11 & 13 & 16 & 5 & 12 \\
\hline Pays de Galles & 4 & 4 & 6 & 13 & 12 & 20 & 5 & 13 & 3 & 4 & 5 & 11 & 11 & 15 & 4 & 11 \\
\hline Écosse & 2 & 3 & 5 & 11 & 12 & 20 & 4 & 13 & 3 & 4 & 5 & 10 & 12 & 15 & 4 & 11 \\
\hline
\end{tabular}

Source : tableau établi par l'auteur d'après Census 1981 et Census 1997, via Nomis, 2001.

12 Tout d'abord, à la lecture du tableau, on voit qu'en 1981 et en 1991, quelle que soit la région, dans le Sud comme dans le Nord, plus on descend dans la pyramide sociale plus le taux de chômage augmente. On voit également que la principale différence entre le Nord et le Sud tient aux disparités des taux de chômage entre les catégories les moins qualifiées. En effet, en 1981 par exemple, période caractérisée par une forte disparité Nord-Sud, il convient de le rappeler, le taux de chômage des travailleurs non qualifiés était de $11 \%$ dans le Sud contre $18 \%$ dans le Nord, alors que les taux de chômage des classes situées en haut de la hiérarchie sociale ne variaient guère d'une partie à l'autre du pays. De même, si on compare la situation des travailleurs manuels et non manuels, on constate que l'inégalité Nord-Sud fut nettement plus lisible parmi les premiers. Le taux de chômage pour ce groupe s'établissait à $13 \%$ dans le Nord contre seulement $8 \%$ au Sud. Pour les travailleurs non manuels, le taux de chômage dans la moitié nord et la moitié sud du pays étaient similaires : $4 \%$ au Nord et $3 \%$ au Sud.

Dix ans plus tard, la similitude des taux de chômage régionaux entre les classes sociales les plus qualifiées s'est maintenue. En revanche, on remarque un rapprochement sensible entre le Nord et le Sud du taux de chômage des travailleurs les moins qualifiés. En effet, alors que le taux de chômage des travailleurs non qualifiés par exemple, affiche une baisse non négligeable dans le Nord, passant de $18 \%$ à $14 \%$, dans le Sud de la GrandeBretagne le taux pour ce groupe accuse une légère hausse, passant de $11 \%$ à $12 \%$. De même, le taux de chômage des travailleurs manuels baisse dans le Nord, alors que dans le Sud, le fléau progresse légèrement. Donc ce qui a contribué à atténuer les disparités régionales, ce ne fut pas l'évolution des différences entre les classes les plus qualifiées, mais l'amélioration dans les régions du Nord de la situation des classes les moins qualifiées.

14 Comment expliquer la baisse relative du chômage des "cols bleus » dans les régions jusque-là les plus durement touchées? Si on examine l'évolution de l'emploi, force est de constater que l'explication ne repose nullement sur un dynamisme retrouvé de l'emploi manuel ou peu qualifié. Au contraire, à la lecture du tableau 3, on constate que l'emploi manuel a même baissé ; déclin qui en outre fut plus important au Nord qu'au Sud. 
Tableau 3. Progression de l'emploi par classe sociale, pour les travailleurs manuels et non manuels, 1981-1991

\begin{tabular}{|c|c|c|c|c|c|c|c|c|}
\hline & & & & & & \multicolumn{3}{|r|}{ En \% } \\
\hline & \multicolumn{5}{|c|}{ Classe Sociale } & \multirow[b]{2}{*}{$\mathrm{V}$} & \multirow[b]{2}{*}{ Non Manuel } & \multirow[b]{2}{*}{ Manuel } \\
\hline & I & II & IIIN & IIIM & IV & & & \\
\hline Grande-Bretagne & 23 & 36 & -3 & -13 & -17 & 6 & 16 & -12 \\
\hline "Le Sud" & 25 & 40 & -3 & -11 & -15 & 12 & 18 & -10 \\
\hline "Le Nord" & 21 & 31 & -3 & -15 & -18 & 1 & 13 & -14 \\
\hline Sud-Est & 21 & 39 & -8 & -14 & -21 & 5 & 14 & -14 \\
\hline Est Anglie & 45 & 49 & 13 & -1 & -5 & 30 & 32 & 1 \\
\hline Sud-Ouest & 28 & 41 & 10 & -3 & -4 & 21 & 25 & -1 \\
\hline Midlands de l'Est & 35 & 45 & 3 & -12 & -9 & 20 & 24 & -8 \\
\hline Midlands de l'Ouest & 23 & 39 & -3 & -10 & -13 & 2 & 16 & -10 \\
\hline Yorkshire \& Humberside & 27 & 32 & -1 & -16 & -17 & 6 & 15 & -14 \\
\hline Nord-Ouest & 16 & 29 & -6 & -16 & -24 & -8 & 10 & -18 \\
\hline Nord & 21 & 27 & -4 & -19 & -17 & -1 & 11 & -16 \\
\hline Pays de Galles & 15 & 25 & 4 & -12 & -8 & 5 & 15 & -8 \\
\hline Écosse & 21 & 31 & -3 & -17 & -22 & 7 & 13 & -16 \\
\hline
\end{tabular}

Source : tableau établi par l'auteur d'après Census 1987 et Census 1997, via Nomis, 2001.

Le déclin de l'emploi manuel, au Nord comme au Sud, n'est pas étonnant. En GrandeBretagne, comme d'ailleurs dans la plupart des pays industrialisés, la demande de travailleurs manuels est en nette diminution depuis plusieurs années. À l'origine de cette baisse, on peut mettre en avant deux facteurs : d'une part, le progrès technologique, et d'autre part, la concurrence des pays en voie de développement dans la production des biens manufacturés. ${ }^{3}$

16 Que ce phénomène ait entraîné une plus grande perte d'emplois manuels et peu qualifiés au nord de la Grande-Bretagne qu'au sud du pays n'a rien de surprenant car le bastion traditionnel des « cols bleus » se trouve effectivement dans le Nord. Cependant, observer que le chômage y a en même temps diminué est bien plus étonnant.

Pour y voir plus clair, il convient d'examiner, non pas l'évolution de la demande de travail peu qualifié, mais l'évolution de l'offre. Les rapports entre l'emploi, le chômage et la population active s'appuient sur des dynamiques régionales très différentes. Une baisse d'emploi identique se traduira ici par plus de chômage, là par une émigration plus importante, ailleurs par une « retraite » de la vie active. Un chômage à la baisse peut donc aussi refléter en réalité, une diminution en ressource de la main-d'œuvre.

18 En effet, si on observe l'évolution de la population active, par classe sociale et pour la catégorie des travailleurs manuels, on constate que c'est effectivement dans la moitié nord de la Grande-Bretagne que la baisse des actifs les moins qualifiés et des travailleurs manuels a été la plus importante. Comme le montre le tableau 4, la population active dans cette catégorie a baissé de $16 \%$ dans le Nord contre une diminution de $8 \%$ dans le Sud ; et la proportion de travailleurs non qualifiés a même été à la hausse au Sud, enregistrant une croissance de $13 \%$, alors que dans le même temps au Nord, cette catégorie socioprofessionnelle a accusé une légère diminution de $3 \%$. 
Tableau 4. Évolution de la population active par classe sociale et pour les travailleurs manuels et non manuels, 1981-1991

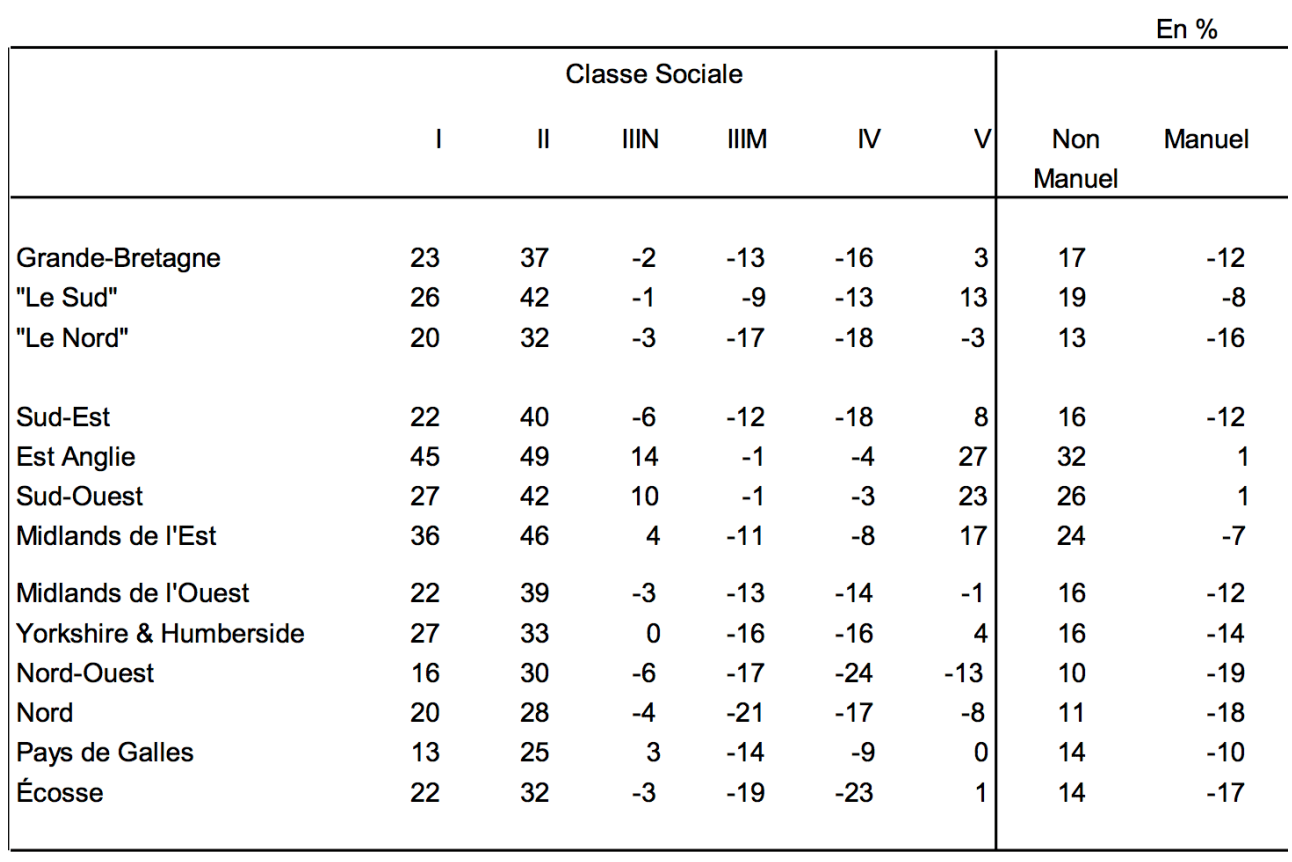

Source : tableau établi par l'auteur d'après Census 1981 et Census 1991 via Nomis, 2001.

19 En somme, sans cette évolution démographique différenciée de la ressource en maind'œuvre peu qualifiée, entre le Nord et le Sud, l'atténuation des disparités des taux de chômage régionaux aurait sans doute été moins apparente.

\section{Le chômage de longue durée}

20 Si l'incidence du chômage est en soi un problème majeur, auquel sont confrontées les régions, c'est le chômage de longue durée qui entraîne le plus de difficultés. Lorsque le chômage est de courte durée, de quelques mois seulement, de toute évidence ses coûts économiques et sociaux sont moindres que lorsque la durée du chômage s'allonge. La probabilité de retrouver un emploi diminue rapidement au fur et à mesure que la durée du chômage augmente. Cette situation constitue dans bien des cas le prélude à l'exclusion sociale et à celle du monde du travail.

21 Les raisons sont connues : elles tiennent à la fois aux comportements des demandeurs d'emploi et à l'attitude des employeurs. Du côté des employeurs d'abord, les recherches ont mis en évidence le fait que ceux-ci tendent à interpréter la durée du chômage comme un indicateur négatif des aptitudes du travailleur, et donc hésitent à embaucher un chômeur de longue durée. ${ }^{4} \mathrm{Du}$ côté des demandeurs d'emploi ensuite, les enquêtes montrent qu'à mesure que la durée du temps passé au chômage s'élève, la motivation, le moral et les efforts à faire pour retrouver un emploi diminuent. ${ }^{5}$ Une longue période sans travail aboutit dans nombre de cas à une dégradation des compétences professionnelles. Si les disparités régionales du taux de chômage se sont affaiblies au cours des années 1990, quelle est la place du chômage de longue durée dans cet état de fait ?

22 Tout d'abord, à la lecture du tableau 5 , on observe une hausse notable du chômage de longue durée pour l'ensemble de la Grande-Bretagne. La proportion de personnes au 
chômage depuis un an et plus par rapport au nombre total de chômeurs passe de $23 \%$ en 1980 à $37 \%$ environ en 1995. Sur le plan régional, on voit que si la montée du chômage de longue durée n'a épargné aucune région, c'est dans le sud du pays que sa progression a été la plus forte, et plus particulièrement dans le Sud-Est où la situation s'est sensiblement dégradée. Comme nous pouvons le constater, le pourcentage des chômeurs de longue durée dans le Sud-Est a plus que doublé en quinze ans, passant de $18,8 \%$ à $38,4 \%$. Ce mauvais résultat fait que, si en 1980 le Sud-Est est la région qui compte le moins de chômeurs de longue durée, quinze ans plus tard, elle devient l'une des régions qui en compte le plus. Cette hausse relativement plus importante dans le sud du pays et la progression relativement moins sensible dans le Nord font que le clivage Nord-Sud du chômage de longue durée ne correspond plus à une réalité géographique.

Tableau 5. Évolution du chômage de longue durée (1 an et plus)

\begin{tabular}{|c|c|c|c|c|c|c|c|c|c|c|c|c|c|c|c|c|c|}
\hline \multicolumn{18}{|c|}{ En \% du chômage total } \\
\hline & 1980 & 1981 & 1982 & 1983 & 1984 & 1985 & 1986 & 1987 & 1988 & 1989 & 1990 & 1991 & 1992 & 1993 & 1994 & 1995 & $\begin{array}{l}\text { Taux de } \\
\text { progression } \\
1980-1995\end{array}$ \\
\hline Grande-Bretagne & 23,0 & 20,1 & 32,8 & 35,8 & 38,9 & 40,4 & 40,5 & 41,3 & 40,0 & 38,6 & 31,9 & 24,1 & 29,9 & 35,0 & 37,6 & 36,6 & 59,4 \\
\hline Sud-Est & 18,8 & 14,7 & 27,1 & 30,1 & 33,6 & 35,7 & 36,3 & 37,7 & 37,6 & 35,5 & 26,5 & 18,8 & 27,1 & 34,4 & 38,3 & 38,4 & 103,8 \\
\hline Est Anglie & 19,6 & 16,5 & 27,3 & 30,1 & 33,2 & 34,1 & 33,5 & 34,4 & 34,1 & 31,4 & 21,5 & 17,3 & 24,4 & 29,7 & 32,9 & 31,0 & 58,1 \\
\hline Sud-Ouest & 23,7 & 19,1 & 29,7 & 31,2 & 32,1 & 33,7 & 33,9 & 34,3 & 32,6 & 31,8 & 24,5 & 17,4 & 26,2 & 32,7 & 35,0 & 33,1 & 39,8 \\
\hline Midlands de l'Est & 22,8 & 19,5 & 32,9 & 34,8 & 36,4 & 38,9 & 40,1 & 39,6 & 39,6 & 39,7 & 29,1 & 23,1 & 29,5 & 34,7 & 37,1 & 35,8 & 57,4 \\
\hline "Le Sud" & 20,5 & 16,4 & 28,4 & 31,0 & 33,8 & 35,8 & 36,4 & 37,3 & 36,9 & 35,4 & 26,3 & 19,1 & 27,1 & 33,9 & 37,4 & 36,8 & 79,9 \\
\hline Midlands de l'Ouest & 24,2 & 20,1 & 38,2 & 42,1 & 46,3 & 47,1 & 46,6 & 47,1 & 45,5 & 43,6 & 35,1 & 25,3 & 31,8 & 38,5 & 42,2 & 40,8 & 68,7 \\
\hline Yorkshire \& Humberside & 22,5 & 20,3 & 34,2 & 36,6 & 39,6 & 41,6 & 39,9 & 43,4 & 41,6 & 40,2 & 33,6 & 26,4 & 32,5 & 36,0 & 37,0 & 35,2 & 56,1 \\
\hline Nord-Ouest & 26,1 & 23,6 & 35,4 & 39,1 & 43,1 & 44,7 & 44,9 & 45,6 & 42,6 & 40,9 & 36,9 & 30,3 & 33,6 & 37,2 & 38,0 & 36,0 & 37,9 \\
\hline Nord & 25,5 & 25,6 & 37,8 & 40,5 & 43,2 & 45,1 & 45,0 & 44,8 & 42,3 & 39,7 & 35,8 & 29,1 & 34,0 & 36,0 & 36,8 & 37,7 & 47,6 \\
\hline Pay de Galles & 23,3 & 22,2 & 34,8 & 37,7 & 40,7 & 42,1 & 41,5 & 41,2 & 37,2 & 35,4 & 29,2 & 23,2 & 31,4 & 35,2 & 36,3 & 34,6 & 48,5 \\
\hline Écosse & 23,3 & 23,2 & 33,0 & 36,7 & 39,3 & 39,9 & 41,0 & 40,4 & 40,1 & 40,0 & 37,4 & 31,3 & 32,6 & 34,6 & 34,3 & 33,9 & 45,4 \\
\hline "le Nord" & 24,3 & 22,4 & 35,6 & 38,9 & 42,2 & 43,5 & 43,3 & 43,9 & 41,9 & 40,3 & 35,3 & 28,1 & 32,7 & 36,2 & 37,8 & 36,4 & 49,5 \\
\hline
\end{tabular}

Source : tableau établi par l'auteur d'après Employment Gazette, via Nomis, 2001.

23 Pour mieux comprendre les dynamiques qui expliquent cette nouvelle configuration, une analyse du chômage en termes de flux d'entrée dans le chômage et de sortie du chômage, peut donner des éclairages supplémentaires. Jusqu'à présent, nous avons analysé le chômage en termes de "stock ", c'est-à-dire de volume global du chômage à un moment donné. Le niveau de ce "stock », en effet, dépend de deux mouvements opposés : celui des entrées dans le «stock» ( le taux d'entrée) et celui dans le même temps des sorties du «stock» ( le taux de sortie ). Rappelons que l'on définit par taux d'entrée dans le chômage, le nombre de personnes devenant chômeurs à un moment donné, par rapport à l'ensemble de la population active, et que le taux de sortie du chômage exprime le nombre de personnes cessant d'être au chômage par rapport au nombre total des chômeurs.

Or, au cours des vingt dernières années, les observateurs ont remarqué qu'en GrandeBretagne, comme dans le reste de l'Europe, l'évolution du volume global du chômage dépendait davantage du taux de sortie que du taux d'entrée dans le chômage. En effet, alors que le taux d'entrée est resté stable, le taux de sortie a diminué. ${ }^{6}$ Ainsi la baisse de la probabilité de sortir du chômage a eu comme conséquence l'augmentation de la durée moyenne du chômage, et du chômage de longue durée notamment. Ce constat impliquerait que sur le plan régional, les disparités du taux de chômage, s'expliqueraient aussi, pour une partie au moins, par les différences entre les taux de sortie. L'atténuation du clivage Nord-Sud serait donc étroitement liée à ce fait. 
Si on observe l'évolution du taux d'entrée et du taux de sortie dans le Nord et le Sud, entre 1980 et 1995, cette hypothèse se confirme (voir graphique 3). L'écart du taux d'entrée dans le chômage entre le Nord et le Sud par rapport à la moyenne nationale reste stable, et le risque de devenir chômeur est toujours plus important au Nord qu'au Sud. En revanche, les chances d'en sortir changent radicalement de camp.

Graphique 3. Évolution du taux d'entrée et du taux de sortie du chômage dans le Nord et le Sud, 1980-1995

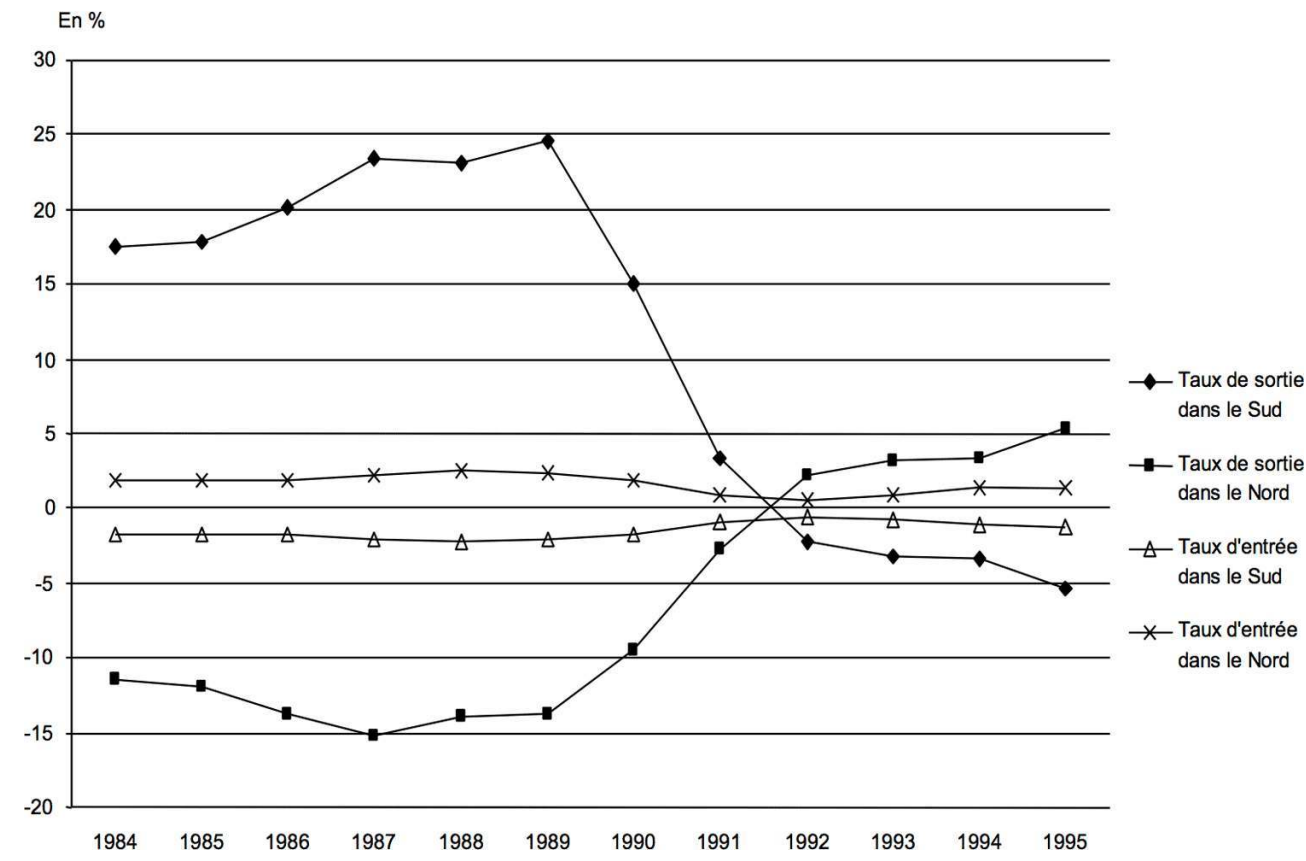

Source : graphique établi par l'auteur d'après Employment Gazette, via Nomis, 2001.

Pour certains chercheurs comme D. Jones et D. Manning, les diverses mesures prises en faveur de la réinsertion professionnelle des chômeurs de longue durée, particulièrement importantes dans les régions les plus touchées, ont sans doute contribué à l'amélioration du taux de sortie dans le Nord. ${ }^{7}$ Mais une autre explication repose sur le fait que si on sort du chômage plus facilement dans le nord c'est peut être parce les chômeurs, au lieu de réintégrer le marché de l'emploi, décident de s'en retirer.

Sur le plan national, ce phénomène, en effet, est en nette progression. Une étude de Paul Gregg et Jonathan Wadsworth, montre que depuis le début des années 1980 en GrandeBretagne, le nombre de chômeurs qui se retirent de la vie active ne cesse d'augmenter. Si au début des années 1980, la proportion de personnes ayant quitté les rangs des chômeurs pour retrouver un emploi s'élevait à environ $75 \%$, au début des années 1990, elles n'étaient plus que $60 \%{ }^{8}$

Sur le plan régional, ce phénomène a été peu étudié, mais on commence à s'y intéresser. À ce sujet, signalons une étude menée par Christina Beatty et Stephen Fothergill, qui ont examiné l'ajustement du marché de travail dans les régions sérieusement touchées par le déclin de l'industrie houillère. Les deux auteurs montrent qu'au cours des années 1980, en dépit des suppressions massives d'emploi dans les bassins houillers, les inscriptions au chômage dans ces régions n'ont pas augmenté. ${ }^{9}$ Outre le facteur migratoire, l'explication principale de cet apparent paradoxe, estiment les deux chercheurs, tient au retrait pur et simple de la vie active. 
29 Cet exemple montre que la prise en considération du phénomène de l'inactivité est indispensable à la compréhension en profondeur de la question des disparités régionales en matière de chômage et d'emploi. En effet, dans une région donnée, si la situation de l'emploi se dégrade mais que les individus quittent le marché du travail pour devenir inactifs, le taux de chômage "visible» peut rester stable, voire être à la baisse. En revanche, si la situation de l'emploi se dégrade mais que les individus restent sur le marché de l'emploi, dans ce cas le chômage augmentera. On peut donc s'interroger sur l'importance du rôle joué par la non-participation à la vie active dans l'atténuation des disparités régionales des taux de chômage.

\section{Le clivage Nord-Sud : le rôle de la participation à la vie active}

30 Une méthode simple qui permet d'évaluer l'importance de ce phénomène consiste à observer pour chaque région l'évolution des taux d'emploi, d'inactivité et de chômage. Signalons ici que ces taux expriment le rapport entre le nombre de personnes dans chacune de ces trois catégories et le nombre total de personnes en âge de travailler; en l'occurrence, de 16 à 64 ans pour les hommes et de 16 à 59 ans pour les femmes. Puisque la différence entre les taux de participation et de chômage est égale au taux d'emploi, on peut évaluer l'importance respective de l'inactivité et du chômage dans les variations de l'emploi. ${ }^{10}$

31 Les résultats de cette analyse montrent qu'entre 1990 et 1995, au Nord, le déclin de l'emploi a effectivement entraîné une hausse bien plus importante de l'inactivité que du chômage recensé (voir tableau 6). En effet, plus de $80 \%$ de la baisse d'emploi s'est traduite par un retrait de la vie active, moins de $20 \%$ donc, a donné lieu à une hausse de chômage. $\mathrm{Au}$ Sud, en revanche, la baisse de l'emploi a occasionné une hausse plus importante du chômage que de l'inactivité. Donc, si le chômage a moins progressé dans le Nord par rapport au Sud, c'est aussi parce que dans le Nord, au lieu de s'inscrire au chômage, une partie plus importante de la population ayant perdu son emploi s'est retirée de la vie active. Pour illustrer ce point, prenons l'exemple du pays de Galles et du Sud-Ouest. Entre 1990 et 1995, la baisse du taux d'emploi fut similaire dans les deux régions. La hausse du chômage en revanche fut sensiblement supérieure dans le Sud-Ouest. Ce qui explique cette différence, c'est le fait qu'au pays de Galles la quasi-totalité des pertes d'emploi, c'est-à-dire presque 90\%, s'est soldée par la hausse de l'inactivé, alors que dans le SudOuest l'inactivité a représenté seulement 30\% de la baisse d'emploi.

Tableau 6. Évolution des taux d'emploi, d'activité et du chômage entre 1990 et 1995.

Image 10000000000004FE000002EEB6BD97AD.png

Source : tableau établi par l'auteur d'après Labour Force Survey, via Nomis, 2001.

Cette observation montre clairement combien il est important de raisonner par rapport à la «trilogie » emploi-chômage-inactivité. Trilogie, par ailleurs, qui n'est pas toujours facile à cerner tant la frontière entre emploi, chômage et inactivité tend à se brouiller. Tout individu peut, en principe, être classé en trois catégories : actif occupé, c'est-à-dire ayant un emploi, inactif ou chômeur. Or ce découpage statistique est loin d'être clairement délimité car des franges importantes de la population se trouvent dans des 
situations qui sont à la lisière de ces trois catégories. À mi-chemin entre l'emploi et le chômage se situe le travail à temps partiel involontaire ; entre l'inactivité et le chômage, on trouve les stages de formation, les dispositifs de préretraite.

Le recours de plus en plus fréquent au travail à temps partiel, la mise en place des dispositifs de préretraite, l'essor des activités de formation professionnelle et de stage pour les demandeurs d'emploi, sont tous des facteurs qui peuvent conduire à déformer la perception des problèmes régionaux. La prise en compte de ces phénomènes permet de nuancer la convergence relative des disparités régionales en Grande-Bretagne.

\section{NOTES}

1. Ian Hamilton FAZEY, Financial Times, 10 September 1990.

2. Voir Anne GREEN, 'The Geography of Changing Female Economic Activity Rates : Issues and Implications for Policy and Methodology', Regional Studies, Vol. 28, n 6, 1994, pp. 639-663.

3. Stephen NICKELL et Brian BELL, 'The Collapse in Demand for the Unskilled and Unemployment across the OECD', Oxford Review of Economic Policy, Vol. 11, n 1, 1995, p. 40.

4. Nigel MEAGER \& Hilary METCALF, Recruitment of the Long-Term Unemployed, London: Institute of Manpower Studies, 1987, p. 140.

5. Richard LAYARD, Stephen NICKELL \& Richard JACKMAN, Unemployment: Macroeconomic Performance and the Labour Market, Oxford: Oxford University Press, 1991, p. 258.

6. R.J. FLANAGAN, 'Labour Market Behaviour and European Economic Growth', in R. LAWRENCE \& C. SCHULTZ (eds.). Barriers to European Growth: A Transatlantic View, Washington DC: Brookings Institute, 1987, p. 195.

7. D. R. JONES \& D.N. MANNING, 'Long Term Unemployment, Hysteresis and the UnemploymentVacancy Relationship: A Regional Analysis', Regional Studies, vol. 26, 1992, p. 22.

8. Paul GREGG \& Jonathan WADSWORTH, 'A Short History of Labour Turnover, Job Tenure and Job Security', Oxford Review of Economic Policy, Vol. 11, n 1, spring 1985, p. 88.

9. Christina BEATTY \& Stephen FOTHERGILL, 'Labour Market Adjustment in Areas of Chronic Industrial Decline: The Case of the UK Coalfields', Regional Studies, Vol. 30, n 7, 1996, p. 627.

10. John SCHMITT \& Jonathan WADSWORTH, 'The Rise in Economic Inactivity', in Andrew GLYN et David MILIBAND (eds.) Paying for Inequality: The Economic Cost of Social Injustice, London: Rivers Oram Press, 1994, p. 233.

\section{RÉSUMÉS}

Au cours des années 1990, on a assisté à une modification profonde des disparités régionales en matière de chômage en Grande-Bretagne. Le clivage traditionnel entre le Nord et le Sud, qui avait tant marqué les années 1980, s'est sensiblement réduit pendant la décennie suivante. Cet article 
examine certaines caractéristiques de la "nouvelle " géographie du chômage en analysant l'évolution du chômage régional par sexe, classe sociale et durée.

During the 1990s, regional unemployment disparities in Great Britain underwent a profound change. The traditional gap between the North and the South, which had widened during the 1980s, began to narrow significantly during the following decade. This article examines some of the characteristics of the 'new' geography of unemployment by giving an analysis of the evolution of regional unemployment by gender, class and duration.

\section{AUTEUR}

\section{VICTOR SARAFIAN}

Université de Lyon 2 